\title{
3D-Sensors for the All-Around Measurement of Teeth, Skin, Face, and Body
}

\author{
Gerd HÄUSLER ${ }^{\star a, b}$, Klaus VEIT ${ }^{a}$

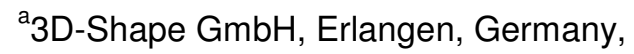 \\ ${ }^{\mathrm{b}}$ Institute of Optics, University Erlangen, Erlangen, Germany
}

\begin{abstract}
In surgery, orthodontics, and dermatology 3D-data sets of soft tissues become more and more stringent. FaceSCAN ${ }^{3 D}$ is a specially designed optical sensor that generates $3 D$-data of human faces for oral and maxillofacial surgery as well as for orthodontics and plastic surgery. The sensor measures fast and accurately the entire face from ear to ear for 3D-visualization for the purpose of surgery planning, study and documentation.
\end{abstract}

Keywords: FaceSCAN ${ }^{3 \mathrm{D}}$, eyeball dislocation, cleft palates, helmet treatment

\section{Introduction}

The commonly used methods for medical data acquisition like radiography and computer tomography expose the patient to radiation. Therefore, it is desirable to introduce alternative non-invasive methods for the data acquisition. For all applications requiring the knowledge of the 3D-geometry of the surface an optical sensor can be employed. Using the fringe projection procedure, bodily contours can be measured accurately and three dimensionally - noses, entire faces, and much more. The measurement results provide important indicators to the surgeons regarding the approach to the operation or to its outcome.

\section{Optical Sensor FaceSCAN ${ }^{3 D}$}

\subsection{The sensor}

The FaceSCAN ${ }^{3 \mathrm{D}}$ sensor [1] depicted in Figure 1 is used for scanning faces and heads, in maxillofacial surgical applications, and for operation planning, for example in jaw orthopedic surgery. The entire face can be scanned from ear to ear (more than $180^{\circ}$ ) with a single shot through an arrangement of mirrors.

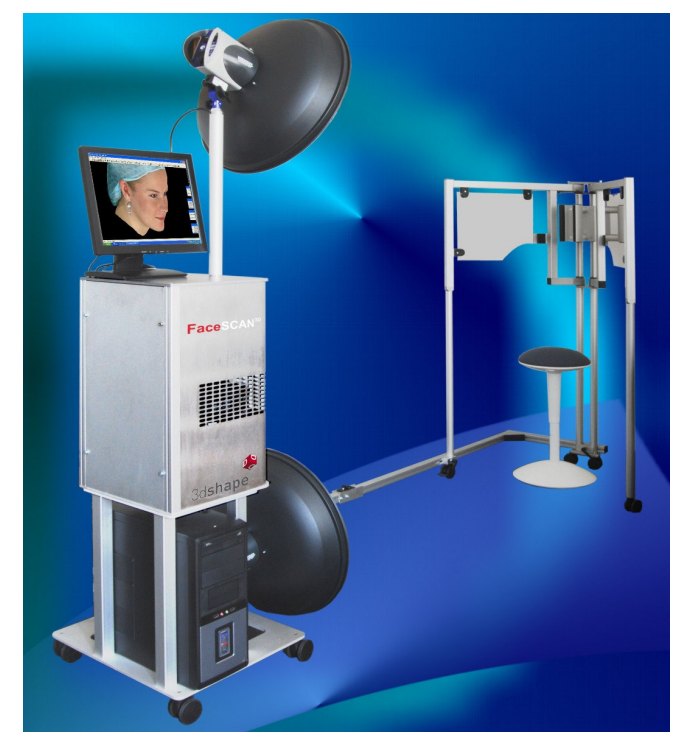

Fig. 1. FaceSCAN $\mathrm{N}^{3 D}$ sensor for measuring a person's head from ear to ear (more than $180 \%$.

*gerd.haeusler@physik.uni-erlangen.de; +49 - 9131 - 8528382; www.optik.uni-erlangen.de/osmin 
The measuring time for one 3D-view is $600 \mathrm{~ms}$ with a measurement uncertainty of about $180 \mu \mathrm{m}$ in case of a measurement field of $600 \times 600 \mathrm{~mm}^{2}$ with a depth range of $360 \mathrm{~mm}$.

Additionally, a high-resolution digital camera captures the surface texture of the face. Software algorithms filter, register, and match the 3D-data and map the color-image onto the data automatically.

\subsection{Method}

The sensor uses a process that illuminates the face with a fringe pattern while two cameras observe the face. The surface shape of the face is calculated using the displacement of the fringes. Fringe projection technology utilizes visible light and fast hardware to carry out measurements of body parts. Its high speed and lack of radiation allow its use with children. Blurring is virtually eliminated through special compensation algorithms [2].

\section{Applications}

In this chapter we present some examples for applications of our FaceSCAN ${ }^{3 \mathrm{D}}$ sensor in the medical field. The selected applications range from eyeball dislocation, over cleft palate, to helmet therapy.

\subsection{Eyeball dislocation}

3D-data are used for treatment planning and to assist surgeons with the correction of an eyeball dislocation. In case of patients with a malposition of the eye globe the position of the displaced eye globe relative to the intact eye globe has to be determined. Using special contact lenses (see Figure 2) we can obtain the 3D-position of the pupil by an optical measurement. Based on the optical 3D-data we developed methods to compute the relative eye globe position as well as the nominal eye globe position.

The goal in the course of this is to place the eyeball precisely in all three dimensions within the eye socket. The same profile sections can be compared with one another before and after the operation using the 3D-data, so that the actual change achieved is precisely quantifiable [3].

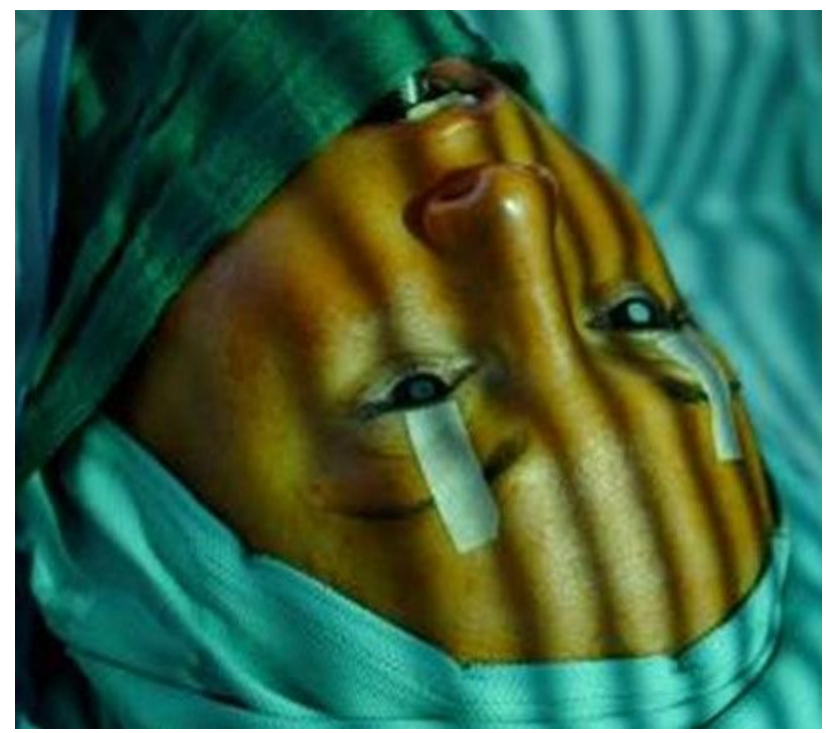

Fig. 2. Patient wearing special contact lenses to capture the 3D-positions of both eye pupils.

\subsection{Cleft palates}

Exact measurements of jaw models can also be carried out in the manner described in the previous section. They deliver the necessary information for the preparation, execution, and long-term follow-up of the treatment of cleft palates. By a point comparison of the physician's coordinates from recording to recording over a period of years a quantifiable process 3D profile can be created. 
Denture castings can also be precisely rendered using the same measurement technique and, among other things, enter as an important parameter into surgical planning: They can be used to simulate profile changes before jaw surgery. In this manner, the 3D-castings deliver crucial data concerning the changeable and unchangeable parts of the face.

\subsection{Helmet treatment}

The heads of infants can show deformations or asymmetries after the birth process. There are many factors that may influence the development of deformational plagiocephaly, such as premature births, restrictive intrauterine positioning, multiple births, and/or birth trauma. The majority of deformations can be treated with physiotherapy - however, a small proportion of deformations persists. To bring the heads to shape a lightweight plastic-helmet can be used [4].

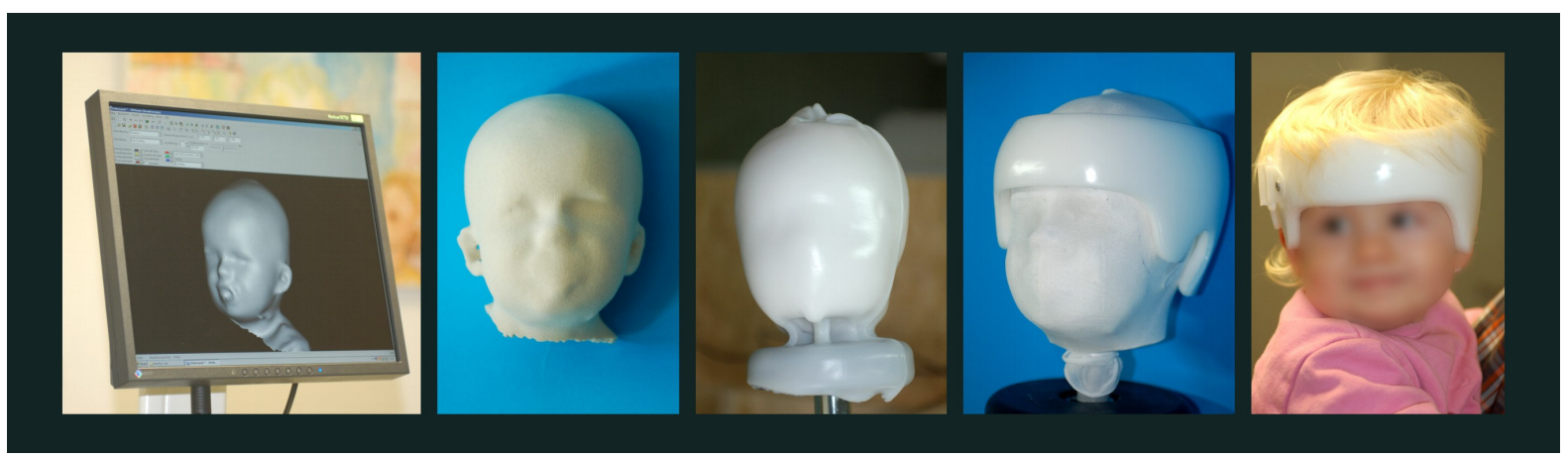

Fig. 3. Helmet treatment for an infant. From left to right: 3D-data captured from the infant using the FaceSCAN ${ }^{3 D}$ sensor. From the 3D-data a 3D-model of the infants' head can be formed. The 3D-model is covered by the basic material of the later helmet. The helmet is obtained by cutting the plastic into the required shape. The lightweight customized helmet fits precisely onto the infant's head.

The helmet is a customized orthosis to treat head shape deformities. At correctly shaped areas the helmet touches the skull, whereas it leaves some space at the deformed areas. The helmet supports the optimal development of the infant's skull, resulting in a well shaped head. The best age for treatment is between 3 and 9 months, because in this time window the skull is growing at its fastest rate.

The FaceSCAN ${ }^{3 D}$ sensor is used for the customization of the helmets by providing the accurate 3D-data of the deformed head (see Figure 3). With the aid of a sophisticated mirror unit and very fast cameras the heads of infants can be scanned all around in a half of a second.

\section{Conclusion}

With our fast optical sensor we obtain precise measurements of the soft tissue surface. Based on this 3D-data we provide the surgeon means for an objective analysis. In the near future, optically measured 3D-surface data will be combinable to CT-data and to cone-beam CT-data. New software tools will increase the number of applications in the medical field. Therefore, it is to be expected that the manifold opportunities of 3D-surface data will find their way to the clinical standard.

\section{References}

1. Face scanning system "FaceSCAN" 3D", (accessed 2010): www.3d-shape.com.

2. Benz, M., Hartmann, J., Maier, T., Nkenke, E., Veit, K., Stellzig-Eisenhauer, A., Neukam, F. W., Häusler, G., (2005): „Optical 3D-metrology for medical applications“. Biomedizinische Technik. Proc. of the jointly held Congresses ICMP 2005 and BMT 2005, pp. 48-49.

3. Nkenke, E., (2005): "3D-Measurement Technology”, Hospital Post, No. 02/2005, p. 11.

4. Helmet treatment (accessed 2010): www.rottkeundmaas.de/index/menuid/113 